Irish Math. Soc. Bulletin

Number 69, Summer/Winter 2012, 63-66

ISSN 0791-5578

\title{
Dana Mackenzie: The Universe in Zero Words, Princeton University Press, 2012, ISBN:978-0-691-15282-0
}

\author{
REVIEWED BY ANTHONY G. O'FARRELL
}

This is a 'concept' book. Elwin Street Productions describe themselves as follows:

We're a lively, independent illustrated coedition publisher. We conceive and produce a mix of stylish reference, handbooks and giftbooks that combine superlative writing and strong concepts with an off-beat sensibility and a fresh, spirited feel.

Their concepts have resulted in a number of series such as The Little Book of $x, x$ in Your Pocket, How to be an $x$, The curious Girl's Book of $x$, Freaky $x, x$ for Busy People (for $x$ here and later substitute a topic such as Climate Change, Conspiracies, Romans, Outer Space, Campfire Cooking, etc.), and many more individual ideas. They conceived the idea of a book about equations, and went looking for a writer. They found Dana Mackenzie, who had established himself as a popular science writer after 13 years as an academic mathematician. He wrote the book, and they placed it with PUP, and so here we are.

There is a recognised need for mathematicians to communicate with the wider public, and there is an appetite out there for digestible material, so although the foregoing scenario does not resemble our usual model (in which we think up the whole idea from the start), I guess it makes sense.

MacKenzie has the knack of getting and keeping your attention, and writes with fluency and wit, and he is a good story-teller. He parses the mathematical universe into Algebra, Geometry, Applied Mathematics and Analysis, and gives each its share. The book is structured into four parts (corresponding to historical periods), each with six chapters. Each chapter has a key equation, ranging from $1+1=2$ to $2^{\aleph_{0}}=\aleph_{1}$, and including physical equations from

Received on 24-8-2012. 
Archimedes, Newton, Maxwell, Einstein and Dirac. This framework provides the skeleton for a tour through the whole history of mathematical ideas and characters, including Pythagoras, Cardano, Kepler, Euler, Abel, Galois, Gauss, Lobachevskii, Hamilton, Riemann and Chern. In the modern period, he includes a chapter on the Lorenz attractor and an account of Black-Scholes and its influence on financial markets. The book is beautifully-illustrated with many full-colour pictures.

There is a wealth of anecdote and information. It was amusing to learn that a 1988 poll taken for the Mathematical Intelligencer revealed that Euler proved four of the top five "most beautiful theorems", and that a similar exercise for Physics World showed Maxwell's Equations to be the most popular.

The author draws morals from the tales, and expresses clear opinions. This makes for interesting reading, although one is obliged to take issue on some points:

He gives a proof by diagram of Pythagoras taken from Liu Hui's third-century annotation of the classic Nine Chapters on the Art of Mathematics, and remarks (p. 39) that it is "a much simpler proof to understand than the one in Euclid's Elements". It is an interesting diagram, but it is not actually a proof, as it implicitly assumes the congruence of various pairs of figures.

I was interested to learn that this same Liu gave the approximation $\pi \approx \frac{3927}{1250}$, based on the use of an equilateral 3072-sided polygon, extending the method used (presumably independently) by Archimedes. On p. 45 he concludes an interesting account of formulae for $\pi$, including one that gives the octal expansion with the quite fatuous statement: "If God created the integers and God created $\pi$, then perhaps God is actually a computer".

On p. 47 he states that "To the modern mathematician, Zeno's paradoxes are harmless". In this, he certainly is in accord with many who underrate Zeno. But Zeno was talking about Physics, the world, and he gave three paradoxes that must be considered together. I would say that they are resolvable, but not that they are harmless.

On p. 51, discussing the area under a parabola, he outlines the method given by Archimedes, and compares it unfavourably with the procedure that would be followed by a "modern mathematician, who would have no qualms about" taking a limit in a certain series. 
But in fact the careful argument used by Archimedes, beginning by assuming that the area is not what is asserted and proceeding by reductio ad absurdum corresponds precisely to what a modern (rigorous) mathematician would do: if the area is not $\frac{4}{3}$, then it is greater or less by some amount $\epsilon>0$, and so on.

Chapter 6, on Archimedes law for levers, includes a statement of his famous Principle about immersed bodies. This Principle is often introduced in school physics courses, as it is here, without any discussion of the reasoning behind it. This is a pity, as it is easily proved in the case of a rectangular box, using elementary arithmetic and the nature of pressure. The case of a body of arbitrary shape requires multivariate integral calculus, or equivalent. It would be inappropriate to include it in the present book, and I do not fault it for that.

On p.73, Kepler's Third Law is mis-stated, using the "distance to the Sun" instead of the semi-axis major. The difference is often trivial enough, but there seems no good reason not to give the correct version. More seriously, there are some questionable statements about the consequences of this Law. It is not true to say that one can infer the distance of an orbiting planet from its period (unless one knows the mass of the star or the distance and period of some other planet). It is not true that one can tell the mass of a planet from the observation of its period - although one can tell it from the period and distance of one of its satellites.

On p. 149, it is implied that the dynamical pressure of the solar wind is the same thing as the pressure of solar radiation.

On p. 185 he gives a proof that the set of real numbers is uncountable, using decimal expansions, remarking in footnote that he "Intentionally gave this easier but flawed version for non-experts. For math experts, repairing the inaccuracy takes a little work but in my opinion no fundamentally new ideas." However, the proof may be fixed in a very simple way without making it any more difficult for non-experts: instead of adding $1 \bmod 10$ to the $n$-th digit of the $n$-th decimal in a purported enumeration of the interval $[0,1)$, just change it to a 2 if it is not a 2 , and otherwise change it to a 3 .

These quibbles are matters that can easily be fixed in a new edition, and once that is done I would be happy to recommend the book to any person, young or old, with an interest in mathematics and its uses. I enjoyed reading it. 
Anthony G. O'Farrell was educated at UCD and Brown University. He is Professor of Mathematics at NUI, Maynooth. His research interests centre on Analysis.

Department of Mathematics and Statistics, NUi, Maynooth, Co. KILDARE

E-mail address: admin@maths.nuim.ie 